\title{
Sevoflurane preconditioning promotes activation of resident CSCs by transplanted BMSCs via miR-210 in a rat model for myocardial infarction
}

\author{
Ti Wen ${ }^{1}$, Li Wang ${ }^{2}$, Xue-Jun Sun ${ }^{3}$, Xi Zhao ${ }^{4}$, Guang-Wei Zhang ${ }^{4}$ and Jesse Li-Ling ${ }^{5}$ \\ ${ }^{1}$ Department of Oncology, The First Hospital of China Medical University, Shenyang, 110001, China \\ ${ }^{2}$ Division of Biotechnology, Dalian Institute of Chemical Physics, Chinese Academy of Sciences, Dalian 116023, China \\ ${ }^{3}$ Department of Anesthesiology, The First Affiliated Hospital of Dalian Medical University, Dalian 116000, China \\ ${ }^{4}$ Department of Cardiac Surgery, The First Hospital of China Medical University, Shenyang, 110001, China \\ ${ }^{5}$ Institute of Genetic Medicine, School of Life Science, State Key Laboratory of Biotherapy, Sichuan University, Chengdu, \\ China \\ Correspondence to: Guang-Wei Zhang, email: lion-roar@163.com \\ Keywords: myocardial infarction; stem cell transplantation; miR-210; regeneration; ventricular function \\ Received: July 29, $2017 \quad$ Accepted: November 14, $2017 \quad$ Published: December 09, 2017 \\ Copyright: Wen et al. This is an open-access article distributed under the terms of the Creative Commons Attribution License 3.0 \\ (CC BY 3.0), which permits unrestricted use, distribution, and reproduction in any medium, provided the original author and source \\ are credited.
}

\section{ABSTRACT}

Objective: To assess the effect of sevoflurane preconditioning (SFpre) on bone marrow mesenchymal stem cells (BMSCs) for the treatment of acute myocardial infarction.

Results: 24 hours after the transplantation, decreased apoptosis of implanted BMSCs and up-regulation of cytokines expression were found within the ischemic area in SFpreBMSCs group compared with BMSCs group $(P<0.05) .4$ weeks later, SFpreBMSCs group showed more viable implanted BMSCs, CSC-derived cardiomyocytes, and higher vessel and myocardial density within the infarcted region and improved cardiac function, compared with control and BMSCs groups $(P<0.05)$. Compared with untreated BMSCs, promoted migration, inhibited apoptosis, increased cytokine secretion, and enhanced activation to CSCs were detected in SFpreBMSCs exposed to profound hypoxia and serum deprivation, via up-regulating miR-210 expression $(P<0.05)$.

Conclusions: Sevoflurane preconditioning can protect BMSCs against hypoxia by activating miR-210 expression and promote their paracrine functions and effects on resident CSCs.

Methods: After the preconditioning, rat BMSCs (SFpreBMSCs group) were transplanted into rat AMI models, while BMSCs group received unconditioned BMSCs. Apoptosis and paracrine functions of the transplanted BMSCs, angiogenesis, resident cardiac stem cells (CSCs) derived myocardial regeneration, cardiac function and remodeling were assessed at various time points. In vitro experiments were performed to determine the expression of miR-210 in BMSCs exposed to sevoflurane and the effect of sevoflurane on BMSCs' migration, apoptosis and secretion of cytokines under hypoxic condition, as well as cytokine-induced CSCs activation. 


\section{INTRODUCTION}

Regenerative therapy for myocardial infarction (MI) has been a great challenge due to the limited potential of myocardial regeneration in adult human heart [1]. Transplantation of bone marrow mesenchymal stem cells (BMSCs) has emerged as a promising method for myocardial regeneration [2-5]. However, this effect has been largely restricted by the limited viability of BMSCs under the severe hypoxic and ischemic condition of the infarcted region, where a complete revascularization is hard to achieve owing to severe and complicated coronary artery disease [6]. Therefore, it is critical to enhance the capacity of BMSCs against hypoxia in order to increase the efficiency of such therapy $[7,8]$.

Sevoflurane, a novel inhaled anesthetics, has been shown to alleviate organic ischemia-reperfusion injury [9-13] and hypoxia-induced cell apoptosis [14-16]. With additional advantages such as definite dosage, ease for administration, rapid-onset and low cost, it has been regarded by many as a new auxiliary therapeutic option for BMSCs transplantation. Our recent in vitro studies suggested that sevoflurane preconditioning (SFpre) may protect BMSCs against hypoxia and improve their therapeutic potentials [17]. However, it is unclear whether this new method may improve the survival of BMSCs transplanted into ischemic cardiac regions and cardiac functions.

To assess the effect of ${ }^{\text {SFre }}$ BMSCs transplantation on myocardial repair following ischemic injury, we chose a rat model of acute MI (AMI) to evaluate the apoptosis and paracrine function of implanted BMSCs, angiogenesis, resident cardiac stem cells-mediated myocardial regeneration, myocardial density in the infarcted areas, and left ventricular (LV) function and remodeling. To explore the underlying mechanism, in vitro experiments were carried out to assess the potential of ${ }^{\text {SFre }}$ BMSCs against hypoxia by determining the expression of miR-210 (an important anti-hypoxic factor [18, 19]) and its target gene caspase 8 associated protein 2 (Casp8ap2) and protein tyrosine phosphatase, non-receptor type 2 (PTPN2), cell migration, apoptosis and secretion of cytokines, as well as the induced activation of cardiac stem cells (CSCs).

\section{RESULTS}

\section{Sevoflurane preconditioning inhibited apoptosis of transplanted BMSCs and enhanced their paracrine function}

As indicated by TUNEL staining (Figure 1A), 24 hours after the operation, fewer apoptotic BMSCs have transplanted into the infarcted regions in the ${ }^{\text {SFpre }}$ BMSCs group $(78.5 \pm 4.27 \%, n=6)$ compared with the BMSCs group $(90.33 \pm 3.14 \%, n=6, P<0.001)$, Figure 1B. RT-PCR and Western blotting (Figure 1C) revealed that the expression levels of VEGF, bFGF and SDF- $1 \alpha$ were significantly higher in the ${ }^{\text {SFre }}$ BMSCs group compared with the BMSCs group $(P<0.05$, Figure $1 \mathrm{D}$ and $1 \mathrm{E})$.

\section{SFpreBMSCs activated resident CSCs and promoted myocardial regeneration}

As shown by frozen tissue sections 4 weeks after the treatment (Figure 2A), more transplanted BMSCs (CM$\mathrm{DiI}^{+}$) have presented in the core of the ischemic region in the ${ }^{\text {SFpre }}$ BMSCs group $(9.4 \pm 0.43$ cells $/ \mathrm{hpf})$ compared with the BMSCs group $(5.52 \pm 0.38$ cells/hpf, $P<0.001$, Figure 2B). The CM-DiI ${ }^{-}$cells were considered as the resident. Compared with the control and BMSCs groups, the resident $\mathrm{c}-\mathrm{kit}^{+} / \mathrm{GATA}^{+}, \mathrm{BrdU}^{+} / \mathrm{c}-\mathrm{kit}^{+}$and $\mathrm{BrdU}^{+} / \mathrm{cTNT}^{+}$ cells were significantly increased in the ${ }^{\text {SFpre }}$ BMSCs group $(P<0.001)$, although an increase also occurred in BMSCs compared with the control group $(P<0.001$, Figure $2 \mathrm{C})$.

\section{Evaluation of angiogenesis, myocardial viability, $L V$ remodeling and function}

Representative photos of anti-vWF staining are shown in Figure 3A, which revealed a significant increase in NV density in ${ }^{\text {SFre }}$ BMSCs group (OD $=5395 \pm 395$ pixels/hpf) compared with the control $(\mathrm{OD}=2337 \pm 127$ pixels/hpf, $P<0.001)$ and BMSCs group $(\mathrm{OD}=4150 \pm 147$ pixels/hpf, $P<0.001)$, although there was also a difference between the latter two groups $(P<0.001$, Figure 3B). Masson trichrome staining (Figure 3A) showed an enhancement of myocardial density in the ${ }^{\text {SFpre }}$ BMSCs group (OD $=63590 \pm 4950$ pixels/hpf, $P<0.001)$ and BMSCs group $(\mathrm{OD}=34980 \pm 2473$ pixels $/ \mathrm{hpf}, P<0.001)$ as compared with the control group (OD $=13689 \pm 1193$ pixels/hpf). However, the increase was more significant in the ${ }^{\mathrm{SF} p r e} \mathrm{BMSC}$ group compared with the BMSCs group $(P<0.001$, Figure 3C).

4 weeks after the treatment, a significant improvement of left ventricular end diastolic dimension (LVDd), left ventricular anterior wall end-diastolic and end-systolic thickness (LVAWTd) and EF was detected in the ${ }^{\text {SFpre }}$ BMSCs group compared with the control and BMSCs groups $(P<0.05)$, although the benefit was more obvious in the BMSCs group compared with the control group $(P<0.05$, Figure 3D-3G).

\section{Sevoflurane influenced the expression of miR-210 and its target gene}

As shown by in vitro results of RT-PCR and Figure 4A, the expression of miR-210 was up-regulated in BMSCs exposed to hypoxia and ${ }^{\text {SFree BMSCs }}(P<0.05$, Figure 4B). More importantly, this increase was more obvious in SFpreBMSCs compared with the BMSCs $(P<0.05)$, suggesting that sevoflurane may enhance the expression of miR-210 under a hypoxic condition. 
Compared with BMSCs, the expression of miR-210 target gene Casp8ap2 and PTPN2 was decreased in ${ }^{\text {SFre BMSCs }}$ under a hypoxic condition $(P<0.001$, Figure 4C and 4D), which could be reversed by anti-miR-210

\section{Sevoflurane improved migration, apoptosis and cytokines secretion of BMSCs by miR-210}

There was a significant improvement of BMSCs migration $(\mathrm{OD}=0.27 \pm 0.03)$ following sevoflurane preconditioning under a hypoxic condition compared with the untreated BMSCs $(\mathrm{OD}=0.15 \pm 0.01, P<0.001$, Figure 5A and 5B). The hypoxia-induced apoptosis of BMSCs was significantly inhibited by sevoflurane preconditioning $(10.20 \pm 1.18 \%$ vs. $17.82 \pm 1.60 \%$ in untreated BMSCs, $P<0.001$, Figure 5A and 5C). And a similar effect was found in the protein secretion of VEGF, bFGF and SDF-1 $\alpha$, as shown by Figure 5D-5F. These changes could be reversed by anti-miR-210 $(P<0.05)$, indicating that miR-210 is involved in the activation of BMSCs induced by sevoflurane.

In vitro proliferation, migration, apoptosis and cardiac differentiation of CSCs influenced by SFpreBMSCs

In vitro proliferation, migration and myocardial differentiation of CSCs were measured to assess the effect of BMSCs. CSCs migration was enhanced by the supernatant derived from normoxia-cultured BMSCs $(\mathrm{OD}=0.19 \pm 0.01)$ compared with the control $(\mathrm{OD}=$ $0.12 \pm 0.01, P<0.001$, Figure 6A and 6C). This effect could be promoted by hypoxia-culture $(\mathrm{OD}=0.27 \pm 0.02$, $P<0.001)$ and further enhanced by hypoxia-culture with sevoflurane preconditioning $(\mathrm{OD}=0.36 \pm 0.01$, $P<0.001)$. Similar effect was observed with CSCs proliferation $(P<0.001$, Figure 6B), hypoxia-induced apoptosis $(P<0.01$, Figure $6 \mathrm{~A}$ and $6 \mathrm{D})$ and cardiac
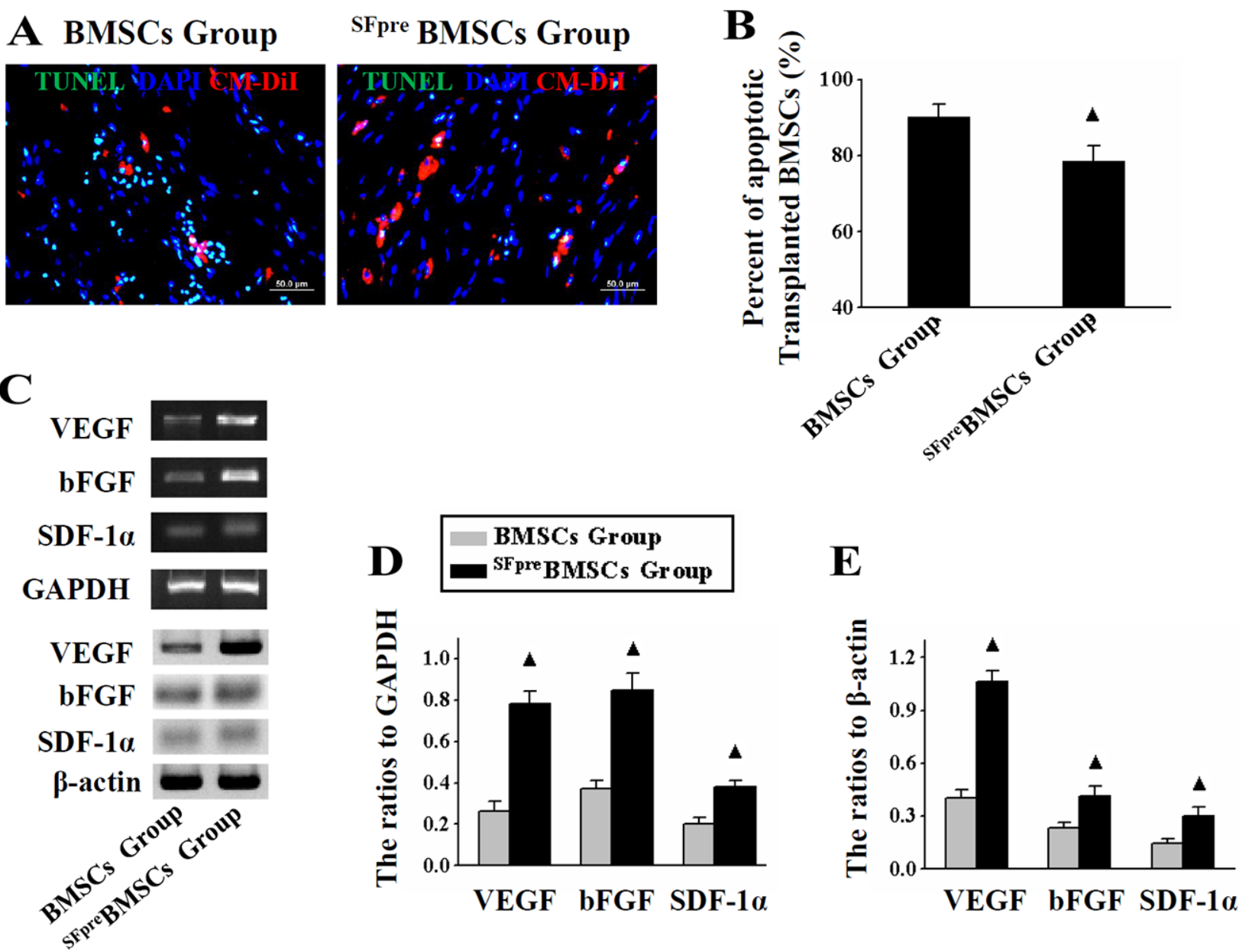

Figure 1: Analysis of apoptosis and paracrine functions of the implanted BMSCs. The representative TUNEL staining

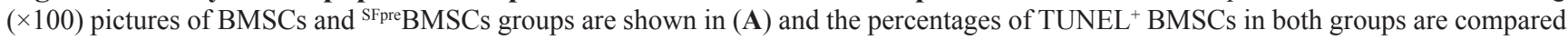
in $(\mathbf{B}, \mathbf{C})$ shows the representative photographs of RT-PCR and Western blotting products in both groups, and (D and E) respectively show the comparison of quantitative analysis results. TUNEL: Terminal deoxynucleotidyl transferase-mediated dUTP nick-end labeling. $\triangle P<0.001$ vs. BMSCs group. 
differentiation $(P<0.05)$, which was shown by the expression of cTNT, a-actinin, and Nkx2.5 as well as anticTNT and anti-CX43 co-staining (Figure 6E-6G).

\section{DISCUSSION}

The present study has demonstrated for the first time that 1) sevoflurane pretreatment can promote the therapeutic effect of BMSCs transplantation on AMI through inhibiting apoptosis, enhancing their paracrine function and activating resident $\mathrm{CSCs}$; 2) sevoflurane pretreatment can inhibit hypoxia-induced BMSCs apoptosis and promote their migration and cytokines secretion through regulating miR-210 and its target genes Casp8ap2 and PTPN2.
BMSCs transplantation can be hampered by the limited survival under ischemic and hypoxic condition of infarcted regions despite their potentials to improve myocardial regeneration and preserve LV function $[2,3,20,21]$. Increasing evidences have suggested that apoptosis of the implanted BMSCs can be largely attributable to hypoxia. Therefore, to enhance the capacity of BMSCs against hypoxia may provide more benefits for such cells to repair myocardial injury $[8,22]$.

Sevoflurane has been demonstrated to confer effective protection for certain organs or cells to against hypoxia [14-16]. Recently, our in vitro studies have shown that this method could promote the therapeutic potential of BMSCs through inhibiting hypoxia- and

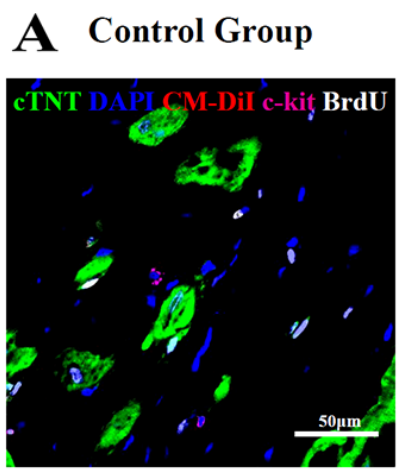

BMSCs Group

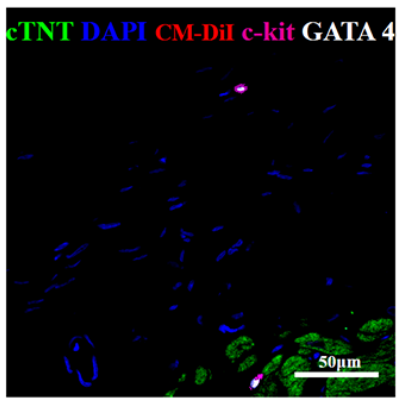

B

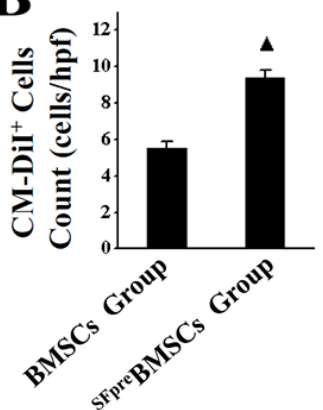

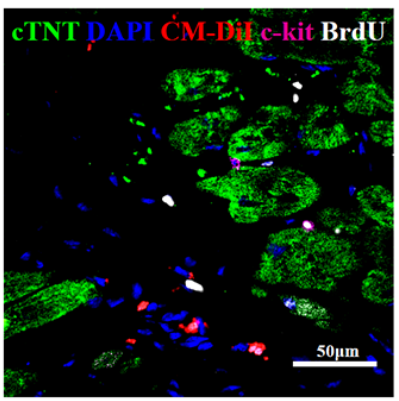
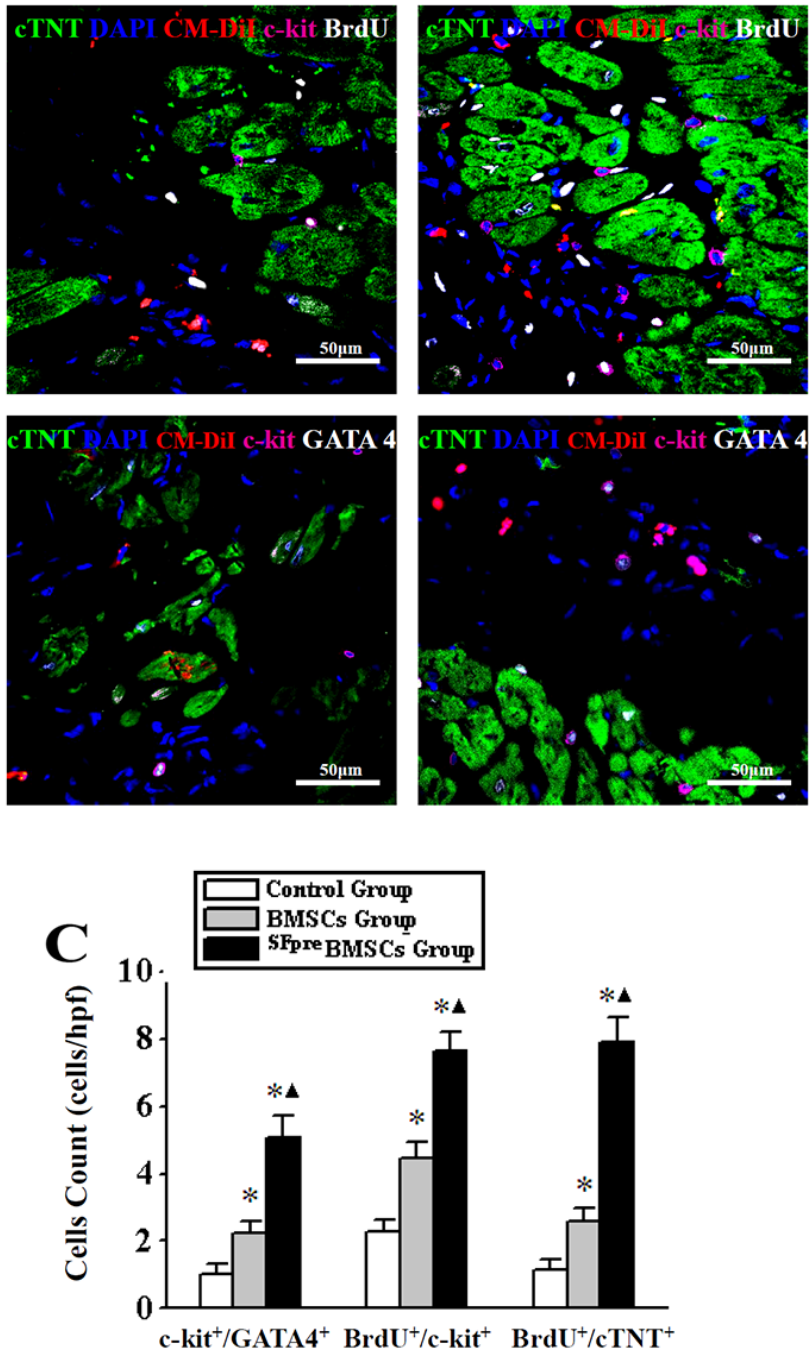

Figure 2: Identification of myocardial regeneration. The representative immunofluorescence staining confocal photos $(\times 600)$ of anti-cTNT (green), DAPI (blue), anti-c-kit (violet), and anti-GATA4 or anti-BrdU (white) are shown in (A). CM-DiI ${ }^{+}$cell (red fluorescence) are conformed as implanted BMSCs and compared in (B), and $\mathrm{CM}_{-} \mathrm{DiI}^{+}$ones were considered as the resident, of which c-kit/GATA4 ${ }^{+}$,

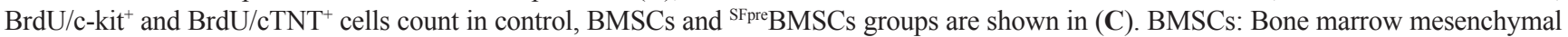
stem cells. BrdU: 5-bromo-2-deoxyuridine. CM-DiI: 1'-dioctadecyl-3,3,3',3'-tetramethylindocar bocyan-ineperchlorate. SFpre: Sevoflurane preconditioning. ${ }^{*} P<0.001$ vs. Control group. ${ }^{\wedge} P<0.001$ vs. BMSCs group. 
serum deprivation-induced apoptosis and promoting the migration and expression of HIF- $1 \alpha$, HIF-2 $\alpha$, VEGF under a hypoxic condition [17]. The present study further confirmed that SFpre can attenuate the apoptosis of the transplanted BMSCs, up-regulate their secretion of VEGF, bFGF and SDF- $1 \alpha$, thereby preserve the cardiac function and suppress ventricular remodeling. Therefore, sevoflurane may provide an auxiliary therapeutic option for cell transplantation.

Paracrine mechanisms may play a crucial role in BMSCs transplantation [8, 23]. Paracrine cytokines from BMSCs including VEGF, bFGF and SDF- $1 \alpha$ have been identified to not only increase angiogenesis and myocardial perfusion, but also promote proliferation, migration and cardiac differentiation of CSCs and decrease hypoxia-induced apoptosis, resulting in promotion of CSCs-mediated myocardial regeneration and improvement of cardiac function [8, 23-26]. Our in vivo and in vitro experiments have revealed that hypoxia can enhance BMSCs' expression of these cytokines, which may be further enhanced by SFpre with a more significant activation of CSCs, which suggested that sevoflurane may also activate paracrine function of BMSCs under normoxia.
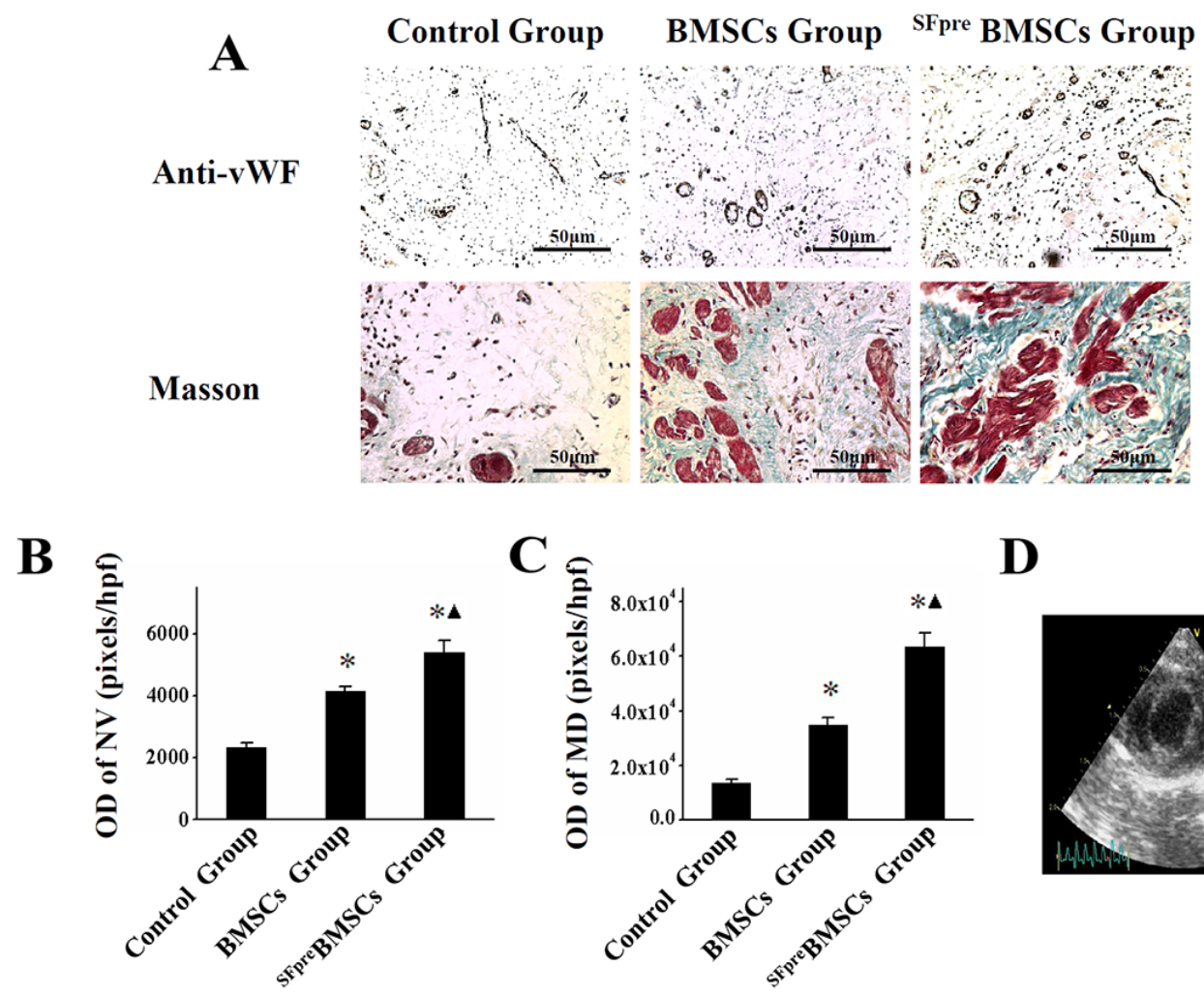

D
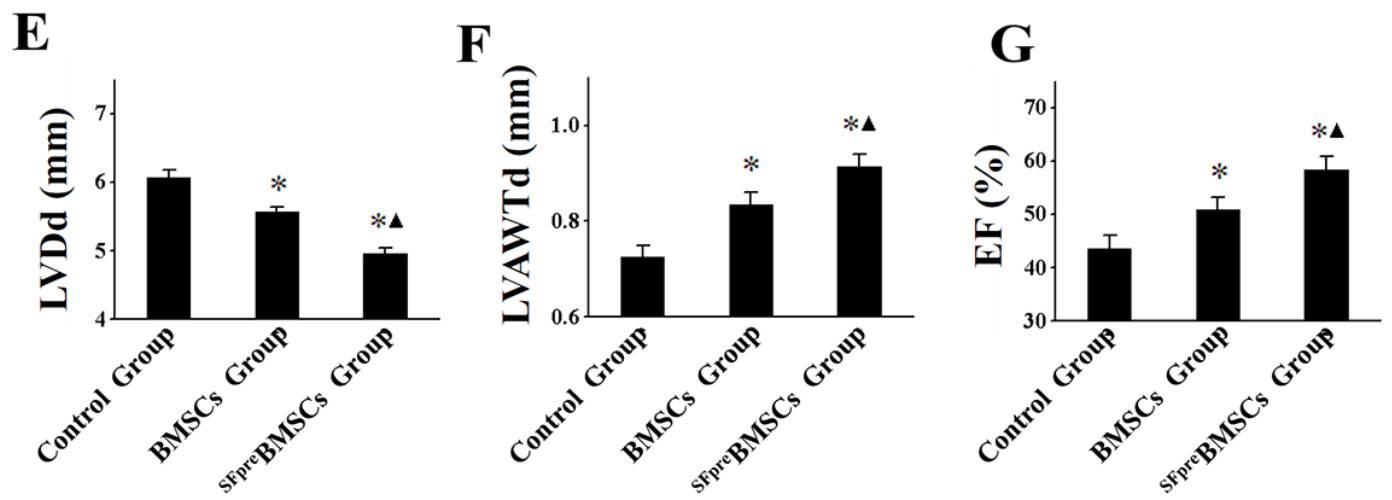

Figure 3: In vivo evaluation of angiogenesis, myocardium density, and LV function. (A) shows the representative photos $(\times 200)$ of anti-vWF staining (top) and Masson trichrome-staining (below: blue indicates collagen; red indicates viable myocardium) in three groups. The quantitative analysis of OD of NV and MD among the three groups is compared in (B and C) respectively. (D) shows the representative picture of echocardiography. $(\mathbf{E}-\mathbf{G})$ respectively display the comparison of LVDd, LVAWTd and EF value. vWF: von Willebrand factor. OD: Optical density. NV: New vessels. MD: Myocardium density. LVDd: left ventricular end diastolic dimension. LVAWTd: left ventricular anterior wall end-diastolic. EF: Ejection fraction. ${ }^{*} P<0.001$ vs. Control group. ${ }^{\wedge} P<0.001$ vs. BMSCs group. 
As an critical regulator of cell anti-hypoxia [18, 27], miR-210 has been identified to contribute to protective mechanisms of SFpre by our in vitro experiments, although other mechanisms may also be involved in such process, including regulation of mitochondrial respiratory function, attenuation of oxidative stress and inhibition of Beclin 1-mediated autophagic cell death [9-11]. As shown by the present study, anti-miR-210 could significantly inhibit SFpre-induced BMSCs activation, which indicates that miR-210 is involved in sevoflurane regulation of BMSCs migration and paracrine besides apoptosis. Caspase-8associated protein 2 (Casp8ap2) and protein tyrosine phosphatase, non-receptor type 2 (PTPN2) have been confirmed as target genes of miR-210 [27, 28], whose expression is decreased in ${ }^{\text {SFpre }} \mathrm{BMSCs}$ under a hypoxic condition and increased after exposure to anti-miR-210, indicating that sevoflurane regulates Casp8ap2 and PTPN2 by miR-210. Base on the evidence that miR-210 inhibited hypoxia-induced apoptosis of stem cells by down-regulation of Casp8ap2 [28], it is speculated that sevoflurane may alleviate the hypoxia-induced apoptosis of BMSCs via miR-210/Casp8ap2 pathway. Additionally, PTPN2 has been documented to regulate the migration of adipose-derived stem cells and cytokine production in bone marrow-derived endothelial precursor cells and hematopoietic stem cells $[27,29,30]$. Therefore, miR-210/PTPN2 pathway may contribute to sevoflurane-induced BMSCs migration under hypoxic condition. Our in vivo and in vitro studies revealed that pretreatment by sevoflurane can promoted the secretion of VEGF, bFGF and SDF- $1 \alpha$ by BMSCs under a hypoxic condition, and that such promotion can be attenuated by anti-miR-210, indicating that sevoflurane may regulate BMSCs paracrine function through miR-210 and its target gene PTPN2, although the larger number of BMSCs protected by sevoflurane may also account for increased cytokine secretion.

Despite the encouraging results, some questions may warrant further research. First, considering the difference between human and rat hearts, our animal experiment may not provide direct guidance for clinical application. Second, there is evidence that other miRNAs may also play a role in the protective mechanism of sevoflurane preconditioning [31-33], which has not been considered in our research. Third, the present study only explored the paracrine effect of transplanted BMSCs on resident CSCs, although other mechanisms may also be involved in BMSCs-mediated cardiac repair. Finally, the effect of various concentrations of sevoflurane were not assessed, therefore the optimum concentration still needs to be determined in the future.

In summary, sevoflurane preconditioning can attenuate the apoptosis of BMSCs transplanted into MI regions and enhance their viability and paracrine function, promote angiogenesis and CSCs-mediated myocardial regeneration, and consequently improve LV remolding and function. The above mechanisms were verified by evaluating the activation of BMSCs under hypoxic condition, as well as the proliferation, migration, and cardiac differentiation of CSCs induced by BMSCs. Additionally, miR-210 may be involved in this protective effect of sevoflurane. This method may provide a new strategy for myocardial regeneration following MI.

\section{MATERIALS AND METHODS}

All animal experiments have been approved by Liaoning Administrative Committee for Laboratory
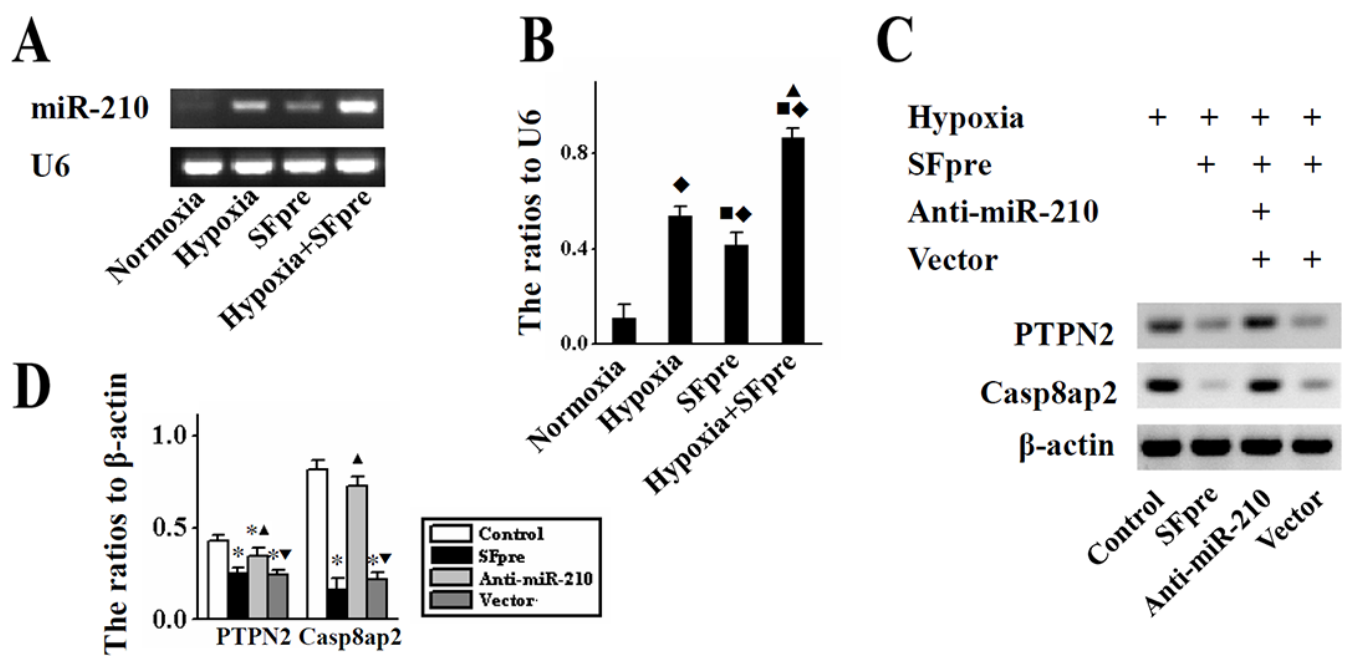

Figure 4: The in vitro expression of miR-210, Casp8ap2 and PTPN2 in BMSCs. Representative photographs of miR-210 expression and the ratio to U6 are shown in (A and B) respectively. The representative Western blotting pictures of Casp8ap2 and PTPN2 are displayed in $(\mathbf{C})$ and the quantitative analysis is performed in (D) Casp8ap2: Caspase 8 associated protein 2. PTPN2: Protein tyrosine phosphatase, non-receptor type 2 . $^{*} P<0.001 v s$. Normoxia. ${ }^{\bullet} P<0.001 v s$. Hypoxia. ${ }^{*} P<0.001 v s$. Control. ${ }^{\mathbf{A}} P<0.001 v s$. SFpre. ${ }^{\mathbf{v}} P<$ 0.001 vs. anti-miR-210. 
Animals and performed strictly according to the "Guide for the Care and Use of Laboratory Animals" published by the National Institutes of Health.

\section{Preparation of BMSCs and CSCs in vitro}

As described in our previous paper [8, 17], BMSCs and CSCs of Sprague-Dawley (SD) rats were isolated and identified. The BMSCs were then labeled with a cross-linkable membrane dye 1'-dioctadecyl-3,3,3',3'tetramethylindocarbocyanine perchlorate (CM-DiI, Invitrogen Corporation, CA, USA) before implantation. To knock down endogenous miR-210, miR-210 inhibitor was transfected to BMSCs with Lipofectamine 2000 (Invitrogen Corporation, CA, USA), followed by a 48 hour incubation. Considering that high concentration $(>3 \%)$ or

\section{A}

$$
\text { Hypoxia }
$$

SFpre

Anti-miR-210

Vector

Migration
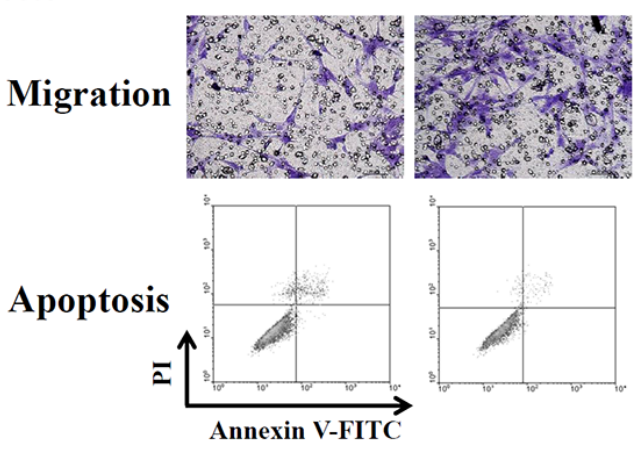

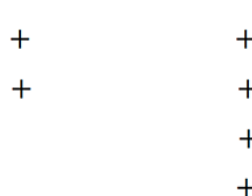

+
+
+
+
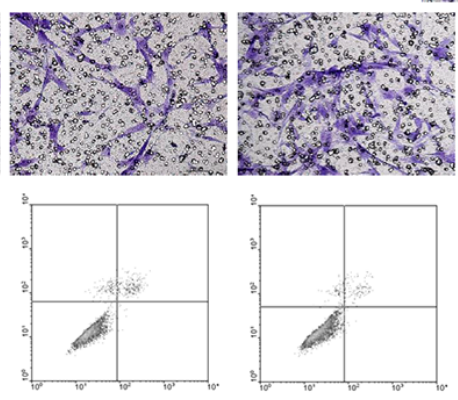

B
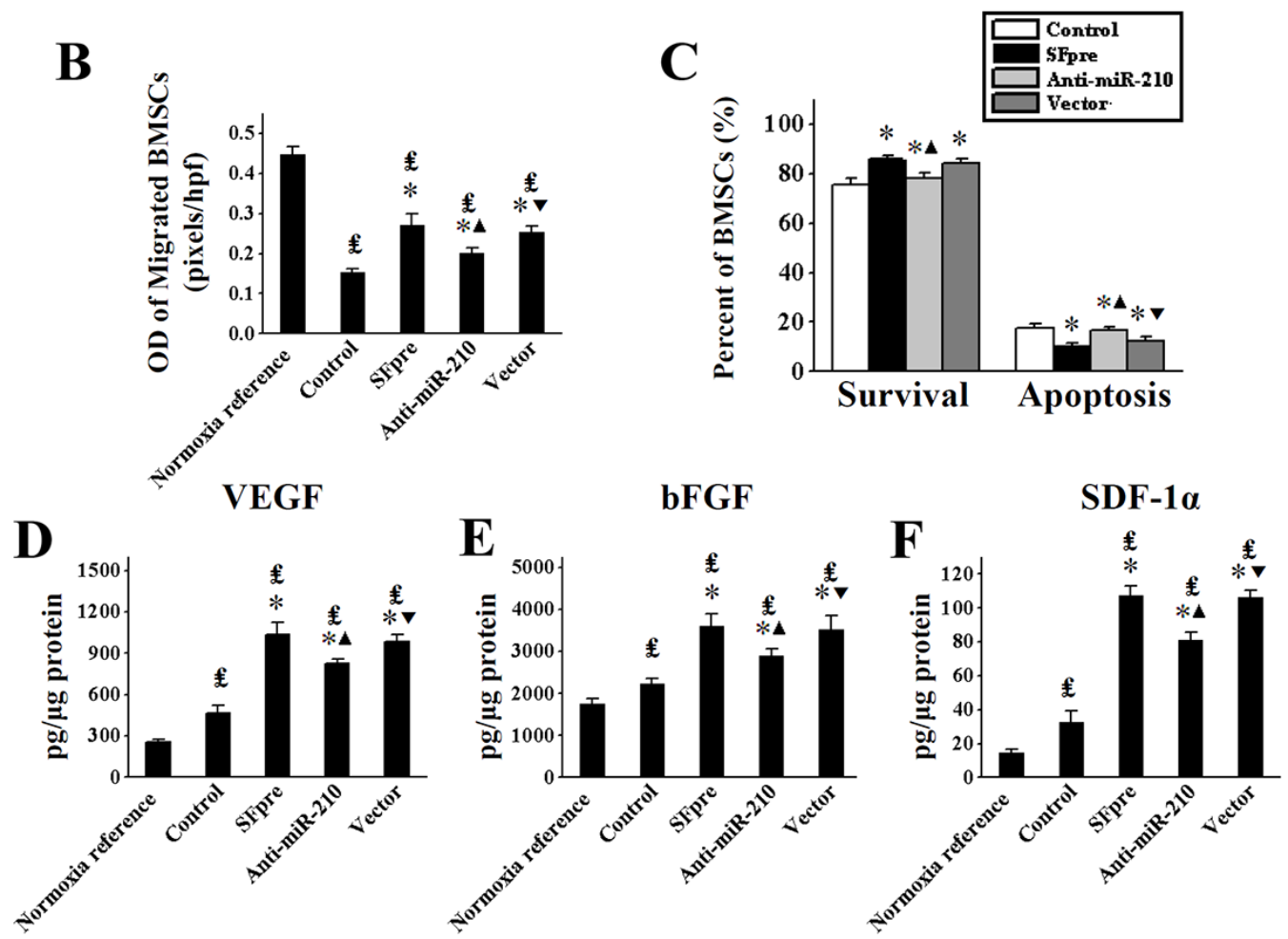

Figure 5: The in vitro assessment of BMSCs activation after exposure to sevoflurane. The images of BMSCs migration (upper row) and Annexin V/PI staining (bottom row) are shown in (A, and comparisons of quantitative analysis for both are exhibited in (B and C) respectively. The comparisons of secreted VEGF, bFGF and SDF-1 $\alpha$ measured by ELISA are respectively exhibited in (D-F) OD: Optical density; VEGF: Vascular endothelial growth factor; bFGF: Basic fibroblast growth factor; SDF-1 $\alpha$ : Stromal cell-derived factor-1 $\alpha$; ELISA: Enzyme-linked immunosorbent assay. ${ }^{\ddagger} P<0.001 v s$. Normoxia reference. ${ }^{*} P<0.001 v s$. Control. ${ }^{\wedge} P<0.001 v s$. SFpre. $\nabla<0.001$ vs. anti-miR-210. 
prolonged exposure ( $>3$ hours) could cause cytotoxicity $[16,34,35]$, a $30 \mathrm{~min}$ exposure of $3 \%$ sevoflurane was chosen to pretreat the BMSCs in the present study.

To imitate the in vivo microenvironment with rather low concentration of oxygen $(0.2 \%$ to $1 \%)$ in the core of the myocardial ischemic region, $4 \times 10^{6} \mathrm{BMSCs}$ or

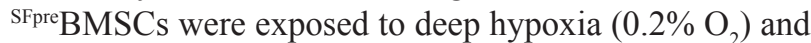
serum deprivation (DH/SD) by using a sealed GENbox hypoxic chamber. Thereafter, the expression of miR-210, Casp8ap2 and PTPN2, cell migration and apoptosis were observed, and the supernatant was harvested for analyzing the cytokine secretion and evaluating their effects on CSCs.

\section{Transplantation of BMSCs}

Adult SD rat (250 300 g) model for AMI was generated by ligating the mid-third of the left anterior descending artery (LAD) as described previously $[8,36]$. Subsequently, the rats were randomly divided into Control
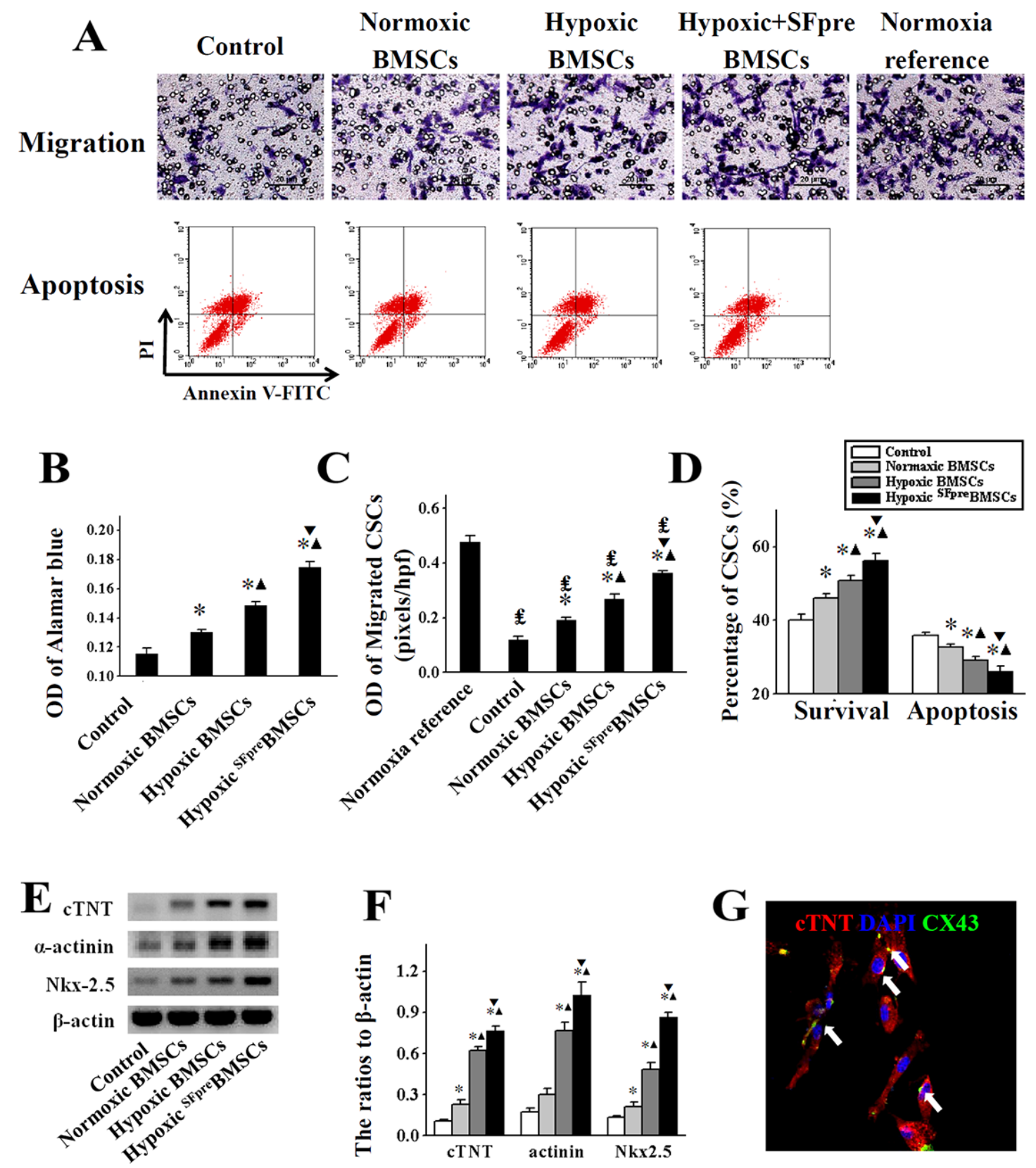

Figure 6: The in vitro evaluation of CSCs activation induced by BMSCs. (A) shows the representative images of CSCs migration (upper row) and apoptosis (bottom row), and comparison of quantitative analysis are respectively exhibited in (C and D). OD values of Alamar blue for determination of proliferation are shown in (B). The representative pictures of Western blotting products for cTNT, $\alpha$-actinin and Nk2.5 are shown in (E) and quantitative analyses were performed in (F) An anti-cTNT (green) and anti-CX43 (red) immunofluorescence staining of CSCs was used to further identify cardiac differentiation in (G) CSCs: Cardiac stem cells; cTNT: Cardiac Troponin T; CX43: connexin 43. ${ }^{*} P<0.001$ vs. Control. ${ }^{\star} P<0.001$ vs. Normoxic BMSCs. $\mathbf{\nabla}<0.001$ vs. Hypoxic BMSCs. 
group (saline injection, $n=10$ ), BMSCs group (untreated BMSCs transplantation, $n=16$ ), and ${ }^{\text {SFpreBMSCs group }}$ (SFpreBMSCs implantation, $n=16$ ). $200 \mu \mathrm{l}$ saline with or without allogeneic $1 \times 10^{7}$ BMSCs was injected into the infarcted area with a sterile microinjection syringe at 2 sites. To label the cells with DNA replication, $50 \mathrm{mg} / \mathrm{kg}$ 5-bromo-2-deoxyuridine (BrdU) was administrated intraperitoneally twice a week postoperatively. The cardiac function and remodeling was assessed by echocardiography.

\section{Proliferation, migration, apoptosis and differentiation of cells}

Proliferation of CSCs was analyzed with an Alamar blue assay (at 1:10 vol/vol ratio, Invitrogen, DAL1025, Carlsbad, CA, USA) with an initial density of 1,000 cells/well in 96-well plates [22] 3 days after exposure to supernatant from BMSCs. The migration of BMSCs and CSCs was assessed with an 8-mum pore-size transwell migration chamber (Millipore, Billerica, MA, USA). The stimuli and cells were respectively added to the lower and upper chambers. Migrated BMSCs (at 8 hours) or CSCs (at 6 hours) were stained with crystal violet, and the absorbance was measured for quantitative analysis. The apoptosis was analyzed with a flow cytometer after annexin $\mathrm{V} /$ propidium iodide staining, after exposure to $\mathrm{DH} / \mathrm{SD}$ for 24 hours for BMSCs or 6 hours for CSCs. The differentiation of CSCs was evaluated by observing the expression of myocardial specific protein, 14 days after culturing in a DMEM/F12 medium with $2 \%$ FBS and supernatant from BMSCs.

\section{RT-PCR, Western blotting and ELISA analysis}

As described previously, the expression of miR210 was determined by RT-PCR with U6 as the internal control. The expression of vascular endothelial growth factor (VEGF), basic fibroblast growth factor (bFGF) and stromal cell-derived factor- $1 \alpha(\mathrm{SDF}-1 \alpha)$ within the tissue sample, Casp8ap2 and PTPN2 within the BMSCs, and Cardiac Troponin T (cTNT), $\alpha$-actinin and Nkx2.5 within the CSCs were respectively measured by Western blotting with $\beta$-Actin as the control. VEGF, bFGF and SDF- $1 \alpha$ secreted by BMSCs into the supernatant were determined with an enzyme-linked immunosorbent (ELISA) assay (R\&D Systems, Minneapolis, MN, USA) according to manufacturer's instruction.

\section{Histological analysis}

Tissue sections was processeded by terminal deoxynucleotidyl transferase-mediated dUTP nick-end labeling (TUNEL) staining for assessing the apoptosis of the transplanted BMSCs, anti-von Willebrand factor (vWF, Abcam Ltd, Cambridge, UK) staining for the evaluation of angiogenesis, Masson trichrome staining to delineate the myofilament structure, as well as anti-cTNT, anti-c-kit, and anti-BrdU staining (Abcam Ltd) to identify CSCs and neonatal cardiomyocytes. Immunofluorescence of lamella of crawling CSCs was performed with anticTNT and anti-connexin43 (CX43) antibodies (Abcam Ltd, Cambridge, UK) to confirm cardiac differentiation. 5 non-overlapping fields in transverse sections of each animal were randomly captured under a light or confocal microscope. Image Pro Plus (IPP) 6.0 software package (IPP, Media Cybernetics, Maryland, USA) were used to determine the myocardial density (MD) and new vessels (NV) through optical density (OD) calibration.

\section{Statistical analysis}

Analysis of all offline results were carried out by investigators blinded to the treatment. Data were presented as mean \pm standard deviation. Independent 2-samples Students $t$ test and one-way analysis of variance (ANOVA) with Bonferroni post hoc correction were carried out with a SPSS 19.0 software package (SPSS Inc, Chicago, USA), to compare measurements in each group. $P<0.05$ indicates the difference is statistically significant.

\section{Abbreviations}

AMI: Acute myocardial infarction; ANOVA: Analysis of variance; bFGF: basic fibroblast growth factor; BrdU: 5-bromo-2-deoxyuridine; BMSCs: Bone marrow mesenchymal stem cells; Casp8ap2: Caspase 8 associated protein 2; CM-DiI,: 1'-dioctadecyl-3,3,3',3'tetramethylindocar bocyan-ineperchlorate; CSCs: Cardiac stem cells; cTnT: Cardiac Troponin T; CX43: Connexin43; DH/SD: Deep hypoxia and serum deprivation; EF: Ejection fraction; ELISA: Enzyme-linked immunosorbent; GAPDH: Glyceraldehyde-3-phosphate dehydrogenase; IPP: Image Pro Plus; LAD: Left anterior descending artery; LV: Left ventricle; MD: Myocardial density; NV: New vessels; OD: Optical density; PTPN2: Protein tyrosine phosphatase, non-receptor type 2; RT-PCR: Reverse transcription polymerase chain reaction; SDF$1 \alpha$ : Stromal cell-derived factor-1 $\alpha$; SD: Sprague-Dawley; SFpre: Sevoflurane preconditioning; SPSS: Statistical Product and Service Solutions; TUNEL: Terminal deoxynucleotidyl transferase-mediated dUTP nick-end labeling; VEGF: Vascular endothelial growth factor.

\section{ACKNOWLEDGMENTS AND FUNDING}

This work was supported by the National Natural Science Foundation of China (No. 81400196), Science and Technology Research Program of the Educational Department of Liaoning Province (No. L2013297, L2013316) and Science and Technology Plan Project of Liaoning Province (No. 2015020734). Technical 
assistance of cardiac surgeons (Yong-Hua Bi, Feng Lyu) is gratefully acknowledged.

\section{CONFLICTS OF INTEREST}

None declared.

\section{REFERENCES}

1. Laflamme MA, Murry CE. Heart regeneration. Nature. 2011; 473:326-335.

2. Schachinger V, Erbs S, Elsasser A, Haberbosch W, Hambrecht R, Holschermann H, Yu J, Corti R, Mathey DG, Hamm CW, Suselbeck T, Assmus B, Tonn $\mathrm{T}$, et al. Intracoronary bone marrow-derived progenitor cells in acute myocardial infarction. N Engl J Med. 2006; 355:1210-1221.

3. Wollert KC, Meyer GP, Lotz J, Ringes-Lichtenberg S, Lippolt P, Breidenbach C, Fichtner S, Korte T, Hornig B, Messinger D, Arseniev L, Hertenstein B, Ganser A, Drexler H. Intracoronary autologous bone-marrow cell transfer after myocardial infarction: the BOOST randomised controlled clinical trial. Lancet. 2004; 364:141-148.

4. Srivastava D, Ivey KN. Potential of stem-cell-based therapies for heart disease. Nature. 2006; 441:1097-1099.

5. Guijarro D, Lebrin M, Lairez O, Bourin P, Piriou N, Pozzo J, Lande G, Berry M, Le Tourneau T, Cussac D, Sensebe L, Gross F, Lamirault G, et al. Intramyocardial transplantation of mesenchymal stromal cells for chronic myocardial ischemia and impaired left ventricular function: Results of the MESAMI 1 pilot trial. Int J Cardiol Heart Vessels. 2016; 209:258-265.

6. Chen J, Baydoun AR, Xu R, Deng L, Liu X, Zhu W, Shi L, Cong $\mathrm{X}, \mathrm{Hu} \mathrm{S}$, Chen $\mathrm{X}$. Lysophosphatidic acid protects mesenchymal stem cells against hypoxia and serum deprivation-induced apoptosis. Stem cells. 2008; 26:135-145.

7. Hu X, Yu SP, Fraser JL, Lu Z, Ogle ME, Wang JA, Wei L. Transplantation of hypoxia-preconditioned mesenchymal stem cells improves infarcted heart function via enhanced survival of implanted cells and angiogenesis. J Thorac Cardiovasc Surg. 2008; 135:799-808.

8. Zhang GW, Gu TX, Sun XJ, Wang C, Qi X, Wang XB, Li-Ling J. Edaravone promotes activation of resident cardiac stem cells by transplanted mesenchymal stem cells in a rat myocardial infarction model. J Thorac Cardiovasc Surg. 2016; 152:570-582.

9. Yang L, Xie P, Wu J, Yu J, Yu T, Wang H, Wang J, Xia Z, Zheng $\mathrm{H}$. Sevoflurane postconditioning improves myocardial mitochondrial respiratory function and reduces myocardial ischemia-reperfusion injury by up-regulating HIF-1. Am J Transl Res. 2016; 8:4415-4424.

10. Yu P, Zhang J, Yu S, Luo Z, Hua F, Yuan L, Zhou Z, Liu Q, Du X, Chen S, Zhang L, Xu G. Protective Effect of Sevoflurane Postconditioning against Cardiac Ischemia/
Reperfusion Injury via Ameliorating Mitochondrial Impairment, Oxidative Stress and Rescuing Autophagic Clearance. PLoS One. 2015; 10:e134666.

11. Xie H, Liu Q, Qiao S, Jiang X, Wang C. Delayed cardioprotection by sevoflurane preconditioning: a novel mechanism via inhibiting Beclin 1-mediated autophagic cell death in cardiac myocytes exposed to hypoxia/ reoxygenation injury. Int J Clin Exp Pathol. 2015; $8: 217-226$.

12. Li X, Luo P, Wang F, Yang Q, Li Y, Zhao M, Wang S, Wang Q, Xiong L. Inhibition of N-myc downstreamregulated gene-2 is involved in an astrocyte-specific neuroprotection induced by sevoflurane preconditioning. Anesthesiology. 2014; 121:549-562.

13. Mikrou A, Kalimeris KA, Lilis I, Papoutsidakis N, Nastos K, Papadaki H, Kostopanagiotou GG, Zarkadis IK. Molecular studies of the immunological effects of the sevoflurane preconditioning in the liver and lung in a rat model of liver ischemia/reperfusion injury. Mol Immunol. 2016; 72:1-8.

14. Popescu M, Munteanu A, Isvoranu G, Suciu L, Pavel B, Marinescu B, Zagrean L. Dynamics of endothelial progenitor cells following sevoflurane preconditioning. Roum Arch Microbiol Immunol. 2011; 70:109-113.

15. Lucchinetti E, Zeisberger SM, Baruscotti I, Wacker J, Feng J, Zaugg K, Dubey R, Zisch AH, Zaugg M. Stem cell-like human endothelial progenitors show enhanced colony-forming capacity after brief sevoflurane exposure: preconditioning of angiogenic cells by volatile anesthetics. Anesth Analg. 2009; 109:1117-1126.

16. Qiu J, Shi P, Mao W, Zhao Y, Liu W, Wang Y. Effect of apoptosis in neural stem cells treated with sevoflurane. BMC Anesthesiol. 2015; 15:25.

17. Sun X, Fang B, Zhao X, Zhang G, Ma H. Preconditioning of mesenchymal stem cells by sevoflurane to improve their therapeutic potential. PLoS One. 2014; 9:e90667.

18. Chang W, Lee CY, Park JH, Park MS, Maeng LS, Yoon CS, Lee MY, Hwang KC, Chung YA. Survival of hypoxic human mesenchymal stem cells is enhanced by a positive feedback loop involving miR-210 and hypoxia-inducible factor 1. J Vet Sci. 2013; 14:69-76.

19. Chan YC, Banerjee J, Choi SY, Sen CK. miR-210: the master hypoxamir. Microcirculation. 2012; 19:215-223.

20. Assmus B, Honold J, Schachinger V, Britten MB, FischerRasokat U, Lehmann R, Teupe C, Pistorius K, Martin H, Abolmaali ND, Tonn T, Dimmeler S, Zeiher AM. Transcoronary transplantation of progenitor cells after myocardial infarction. N Engl J Med. 2006; 355:1222-1232.

21. Uemura R, Xu M, Ahmad N, Ashraf M. Bone marrow stem cells prevent left ventricular remodeling of ischemic heart through paracrine signaling. Circ Res. 2006; 98:1414-1421.

22. Zhang GW, Gu TX, Guan XY, Sun XJ, Jiang DQ, Tang R, Qi X, Li XY. Delayed enrichment for c-kit and inducing cardiac differentiation attenuated protective effects of 
BMSCs' transplantation in pig model of acute myocardial ischemia. Cardiovasc Ther. 2015; 33:184-192.

23. Hatzistergos KE, Quevedo $\mathrm{H}$, Oskouei BN, Hu Q, Feigenbaum GS, Margitich IS, Mazhari R, Boyle AJ, Zambrano JP, Rodriguez JE, Dulce R, Pattany PM, Valdes $\mathrm{D}$, et al. Bone marrow mesenchymal stem cells stimulate cardiac stem cell proliferation and differentiation. Circ Res. 2010; 107:913-922.

24. Kinnaird T, Stabile E, Burnett MS, Lee CW, Barr S, Fuchs S, Epstein SE. Marrow-derived stromal cells express genes encoding a broad spectrum of arteriogenic cytokines and promote in vitro and in vivo arteriogenesis through paracrine mechanisms. Circ Res. 2004; 94:678-685.

25. Kinnaird T, Stabile E, Burnett MS, Shou M, Lee CW, Barr S, Fuchs S, Epstein SE. Local delivery of marrowderived stromal cells augments collateral perfusion through paracrine mechanisms. Circulation. 2004; 109:1543-1549.

26. Miyahara Y, Nagaya N, Kataoka M, Yanagawa B, Tanaka K, Hao H, Ishino K, Ishida H, Shimizu T, Kangawa K, Sano S, Okano T, Kitamura S, Mori H. Monolayered mesenchymal stem cells repair scarred myocardium after myocardial infarction. Nat Med. 2006; 12:459-465.

27. Kim JH, Park SG, Song SY, Kim JK, Sung JH. Reactive oxygen species-responsive miR-210 regulates proliferation and migration of adipose-derived stem cells via PTPN2. Cell death \& disease. 2013; 4:e588.

28. Kim HW, Haider HK, Jiang S, Ashraf M. Ischemic preconditioning augments survival of stem cells via miR210 expression by targeting caspase-8-associated protein 2 . J Biol Chem. 2009; 284:33161-33168.

29. Trop S, Tremblay ML, Bourdeau A. Modulation of bone marrow-derived endothelial progenitor cell activity by protein tyrosine phosphatases. Trends Cardiovasc Med. 2008; 18:180-186.

30. Bourdeau A, Trop S, Doody KM, Dumont DJ, Tremblay ML. Inhibition of $\mathrm{T}$ cell protein tyrosine phosphatase enhances interleukin-18-dependent hematopoietic stem cell expansion. Stem cells. 2013; 31:293-304.

31. Ishikawa M, Tanaka S, Arai M, Genda Y, Sakamoto A. Differences in microRNA changes of healthy rat liver between sevoflurane and propofol anesthesia. Anesthesiology. 2012; 117:1245-1252.

32. Lu Y, Jian MY, Ouyang YB, Han RQ. Changes in Rat Brain MicroRNA Expression Profiles Following Sevoflurane and Propofol Anesthesia. Chin Med J. 2015; 128:1510-1515.

33. Morita T, Ishikawa M, Sakamoto A. Identical MicroRNAs Regulate Liver Protection during Anaesthetic and Ischemic Preconditioning in Rats: An animal study. PLoS One. 2015; 10:e0125866.

34. Zhou X, Li YQ, He W, Yang XY, Song FH, Zhou ZB, Tang Y, Feng X, Zhou LH. Effects of sevoflurane and propofol on cultured bone-marrow mesenchymal stem cells of rats. Int J Clin Pharmacol Ther. 2013; 51:332-337.

35. Zhang Y, Dong Y, Zheng H, Shie V, Wang H, Busscher JJ, Yue Y, Xu Z, Xie Z. Sevoflurane inhibits neurogenesis and the Wnt-catenin signaling pathway in mouse neural progenitor cells. Curr Mol Med. 2013; 13:1446-1454.

36. Riemenschneider SB, Mattia DJ, Wendel JS, Schaefer JA, Ye L, Guzman PA, Tranquillo RT. Inosculation and perfusion of pre-vascularized tissue patches containing aligned human microvessels after myocardial infarction. Biomaterials. 2016; 97:51-61. 